Review

\title{
Applications of Random Nonlinear Photonic Crystals Based on Strontium Tetraborate
}

Aleksandr S. Aleksandrovsky ${ }^{1,2, *}$, Andrey M. Vyunishev ${ }^{1,2}$ and Alexandre I. Zaitsev ${ }^{1,2}$

1 L. V. Kirensky Institute of Physics, Akademgorodok, Krasnoyarsk, Russia;

E-Mails: vyunishev@iph.krasn.ru (A.M.V.); az@iph.krasn.ru (A.I.Z.)

2 Siberian Federal University, Svobodnii 79, Krasnoyarsk, Russia

* Author to whom correspondence should be addressed; E-Mail: aleksandrovsky@kirensky.ru; Tel.: +7-391-249-4613; Fax: +7-391-243-8923.

Received: 30 July 2012; in revised form: 12 September 2012 / Accepted: 17 September 2012 /

Published: 1 October 2012

\begin{abstract}
Properties of strontium tetraborate (SBO) and features of as-grown anti-parallel domains are summarized. From the point of view of nonlinear optics, these domains form nonlinear photonic crystals (NPC). Applications of NPC to the deep ultraviolet generation and fs pulse diagnostics are described. NPC and SBO are prospective media for the creation of a widely tunable source of fs pulses in the vacuum ultraviolet and for autocorrelation diagnostics of broadly tunable sources.
\end{abstract}

Keywords: strontium tetraborate; nonlinear photonic crystals; radiation conversion; pulse diagnostics

\section{Introduction}

Spatial modulation of materials' structure or chemical content on a specific scale is known to be a method of strong modification of various macroscopic materials' properties, including mechanical, electric, magnetic, transport and optical ones. The spatial scale of modulation must be chosen in consideration of certain physical phenomena influencing corresponding materials' properties. For instance, nanoscale spatial modulation in crystals leads to the modification of their electronic band structure and a number quantum confinement effects arise from this modulation. In optics, this often leads to the production of material useful for applications, as in case of semiconductor lasers with a quantum well or quantum dot structure of emitting layer. Another example is the spatial modulation of 
the linear optical properties of a material, the first change being within its refractive index. In this case, the interference of electromagnetic waves reflected within the material due to steps of refractive index strongly modifies the spectral dependence of reflection and transmission coefficients. These materials are commonly called photonic crystals (PC) [1]. They are characterized by the optical band structure with band gaps and transmission bands where propagation of waves is either forbidden or allowed. The most common case of these structures is dielectric mirrors and anti-reflection coatings that were extensively investigated long before the concept of photonic crystals was introduced. When luminescent centers are introduced to the photonic crystal then the spectrum of their emission becomes strongly modified, too. The characteristic spatial scale for manifestation of photonic crystal properties is order of wavelength; however, it also depends on the refractive indexes. The small deviation from periodicity in modulation strongly influences some properties of a photonic crystal. The most pronounced case is the photonic crystal with a so-called defect, that is, a single layer with a different thickness or refractive index. If all the structure is properly matched then additional narrow resonances will appear within the band gap. These resonances correspond to localized modes of an electromagnetic field within a PC, the field strength being thousands times higher in the area of defect than outside the crystal. Minor misfit or randomness of the whole structure leads to radical decrease of the field strength within defect.

When linear optical properties of the crystal are not modulated but the nonlinear susceptibility varies alongside the coordinates then this material can be classified as a nonlinear photonic crystal (NPC) [2]. In this case, the power of a nonlinearly generated field from the NPC undergoes modification due to the interference of fields generated by different parts of the material. The spatial scale of nonlinear susceptibility modulation necessary for the manifestation of NPC properties is the inverse wave mismatch for the specific nonlinear optical conversion process. The spatial modulation can be fitted in order to enhance certain nonlinear optical conversion process, as described in the very first paper on nonlinear optics [3]. One can imagine more complex material with simultaneously modulated linear and nonlinear susceptibilities. These media may be either simple linear PC with the single defect nonlinear layer or an arbitrary combination of purely linear and nonlinear layers. Additionally, all these materials are presently classified as NPCs. There are no experimental studies on these structures to date, so in the following we will use the notation NPC for the materials with homogeneous linear susceptibility and modulated nonlinear one, unless opposite is specified.

The simplest case of NPC is 1D structures that were widely known as quasi-phase-matching (QPM) converters long before the emergence of the NPC concept. The main results of studies of QPM converters can be found in [4]. The direction of those studies was governed by the expected application, namely, the nonlinear optical conversion of narrowband $\mathrm{cw}$ laser radiation in the crystalline waveguides with periodically inverted domains. The technology of domain inversion is fairly applicable for ferroelectric crystals, but it is not completely precise and produces domain structures with regular or random deviations from the exact QPM. Therefore, authors of [4] considered the influence of these deviations on the spectral dependence of generated power. This dependence for exact QPM structure with thickness of domains $d_{0}=\pi / \Delta k\left(\lambda_{0}\right)$ is approximately described by $(\sin (x) / x)^{2}$ function, where $x=\left[\Delta k(\lambda)-\Delta k\left(\lambda_{0}\right)\right] d_{0} N / 3.5, N$ being the number of domains. It was shown [4] that in case of special kind of deviations this spectral dependence becomes modified and can be, for instance, a triangular or quasi-rectangular shape. 
During the last decade a new trend in the NPC studies emerged that was connected with the interest to new materials with high degree of randomness in the spatial modulation of nonlinear susceptibility. These materials are mainly ferroelectrics with natural structure of anti-parallel and arbitrary sized domains, or polycrystalline materials. Comprehensive analysis of the most of these studies can be found in several reviews (see, e.g., [5]), so we will mention only main features of random nonlinear media. For such media, a new type of phase matching — random quasi-phase-matching (RQPM) [6], or stochastic QPM [7] — was discovered. It was experimentally demonstrated that in the polycrystalline nonlinear medium consisting of randomly sized and oriented small single crystals, the maximum frequency conversion is attained in the case when the average size of a crystal coincides with the coherence length for the nonlinear conversion process [6]. It was found that while in perfect QPM the power generated in a nonlinear conversion process grows quadratically with the length of the NPC, random nonlinear media demonstrate linear power growth with the length at the scale when medium length exceeds the characteristic randomization length. Random nonlinear crystals were found to be excellent materials for implementation of so-called nonlinear diffraction (NLD), a kind of non-collinear phase matching when wave mismatch is compensated by the reciprocal superlattice vector (RSV) arising from Fourier transform of the nonlinear susceptibility function on the coordinate. Attractiveness of random nonlinear media for nonlinear diffraction is due to the fact that the angle of nonlinear diffraction is determined by refractive indices, while the power of nonlinearly diffracted radiation is determined by Fourier amplitudes corresponding to the RSV required to enable non-collinear phase matching. So, in random media one can find a necessary RSV for a broad range of wavelengths [8] or for arbitrary direction of propagation [9]. As a result, complete conversion of the spectrum of broadband femtosecond pulses in 2D NPC of strontium barium niobate (SBN) crystal was obtained [8]. The spectral dependence of RQPM in the case of strongly randomized crystal was found to be consisting of large number of superimposing peaks [10] that cover the range from near infrared into near UV (on the scale of fundamental wavelengths). Spectral features of both RQPM and random NLD can be briefly described by the statement: "Random media gains tunability for the price of efficiency" [8], that is, efficiency obtainable in a regular structure for narrow bandwidth is distributed over wider spectrum when we have random nonlinear medium.

In recent years, researchers were interested not only in natural random media but also in artificial random media; that is, in the domain structures that contain several superimposed spatial modulation frequencies $[11,12]$. Nonlinear optical properties of such structures must be similar to those of random structures. Most promising material for the formation of artificial random structures is lithium niobate, since artificially formed domains in SBN are shown to be unstable [13].

\section{Orthorhombic Strontium Tetraborate ( $\alpha$-SBO)}

Most of random nonlinear materials investigated up to date are ferroelectrics and formation of random NPC structure in them occurs during the phase transition from paraelectric higher-symmetry phase. The loss of some elements of symmetry group of the unit cell of paraelectric phase is accompanied by formation of anti-parallel domains as if the whole crystal would preserve the symmetry of the paraelectric phase. On the microscopic scale, the process of domain formation at the temperature of phase transition is governed by such factors like defects, impurities etc. As a result, 
domains formed in the ferroelectric phase can be either randomized or highly ordered, depending on degree of microscopic defect randomness.

On the other hand, most ferroelectric materials are not transparent in the ultraviolet, and the results obtained with random media based on them are inapplicable for this spectral range. For instance, most widely studied SBN is transparent down to $400 \mathrm{~nm}$, and the shortest wavelength for lithium tantalate is $270 \mathrm{~nm}$. Nonlinear optical generation in the deep ultraviolet (DUV) and vacuum ultraviolet (VUV) is possible with the help of various borate crystals in the angular phase matching [14], but continuously tunable nonlinear optical conversion to these spectral ranges meets difficulties and limitations. At the same time, some possible applications in industry and science still require new laser sources in DUV/VUV. An attractive possibility is to create powerful femtosecond sources at 193 and $157 \mathrm{~nm}$; this could be implemented using a combination of femtosecond seed pulses at the wavelengths falling into the bandwidths of corresponding excimer molecules and excimer amplification modules. Alternatively, an all-solid-state source at $193 \mathrm{~nm}$ in competition with excimer laser is desirable, and the latter problem was addressed in [15]. The authors of this study managed to create artificial NPC structure in crystalline quartz that is not a ferroelectric but can be mechanically twinned due to favorable symmetry properties of its elastic compliance tensor. Another possible medium for DUV and VUV is orthorhombic strontium tetraborate ( $\alpha$-SBO or simply SBO), which is transparent down to $125 \mathrm{~nm}$ [16]. This crystal belongs to $\mathrm{mm} 2$ point symmetry class, and the crystallographic axes notation used below corresponds to the unit cell parameters $a=4.4145(4) \AA, b=10.6827(10) \AA, c=4.2234(4) \AA$. Nonlinear susceptibility of SBO is the highest among all nonlinear crystals transparent below $270 \mathrm{~nm}$, but birefringence is too small to ensure angular phase matching. Nevertheless, coherent radiation at $125 \mathrm{~nm}$ was generated in this crystal via a non-phase-matched nonlinear frequency doubling, and the possibility for autocorrelation of measurements was demonstrated [16]. SBO is not a ferroelectric, as all other borates. The crystal structure of SBO contains only tetrahedrally coordinated boron ions that form continuous boron-oxygen network. This network seems to be extremely stable against switching of the static polarization direction. The properties of SBO crystal structure and the possibility for inverted domain formation were theoretically investigated in [17]. The crystal structure of SBO was found to be close to certain higher-symmetry structure with two minima for boron atoms. Possible domain inversion would take place in case of displacement of boron atoms from one of these minima to another; however, the energy corresponding to this displacement is in the order of $5000 \mathrm{~K}$.

However, it was found experimentally $[9,18]$ that as-grown boules of SBO obtained via the Czochralski method contain domain structures comprised of anti-parallel domains. An example of such a structure is presented in Figure 1. Compared with known ferroelectric crystals, these domain structures occupy only part of grown crystal. At the same time, the shape and orientation of domains is identical to that of well-known ferroelectric crystal potassium titanyl phosphate (KTP), namely, domains have the form of sheets perpendicular to $a$ crystallographic axis. Static polarization is parallel to $c$ axis, nonzero nonlinear coefficients of SBO are $d_{c a a}, d_{c b b}, d_{c c c}, d_{a a c}$, and $d_{b b c}, d_{c c c}$ being largest of them. 
Figure 1. Domain structure in strontium tetraborate visualized by etching. Crystallographic axes are shown by arrows. Thickness of structure in $a$ direction is $1 \mathrm{~mm}$.

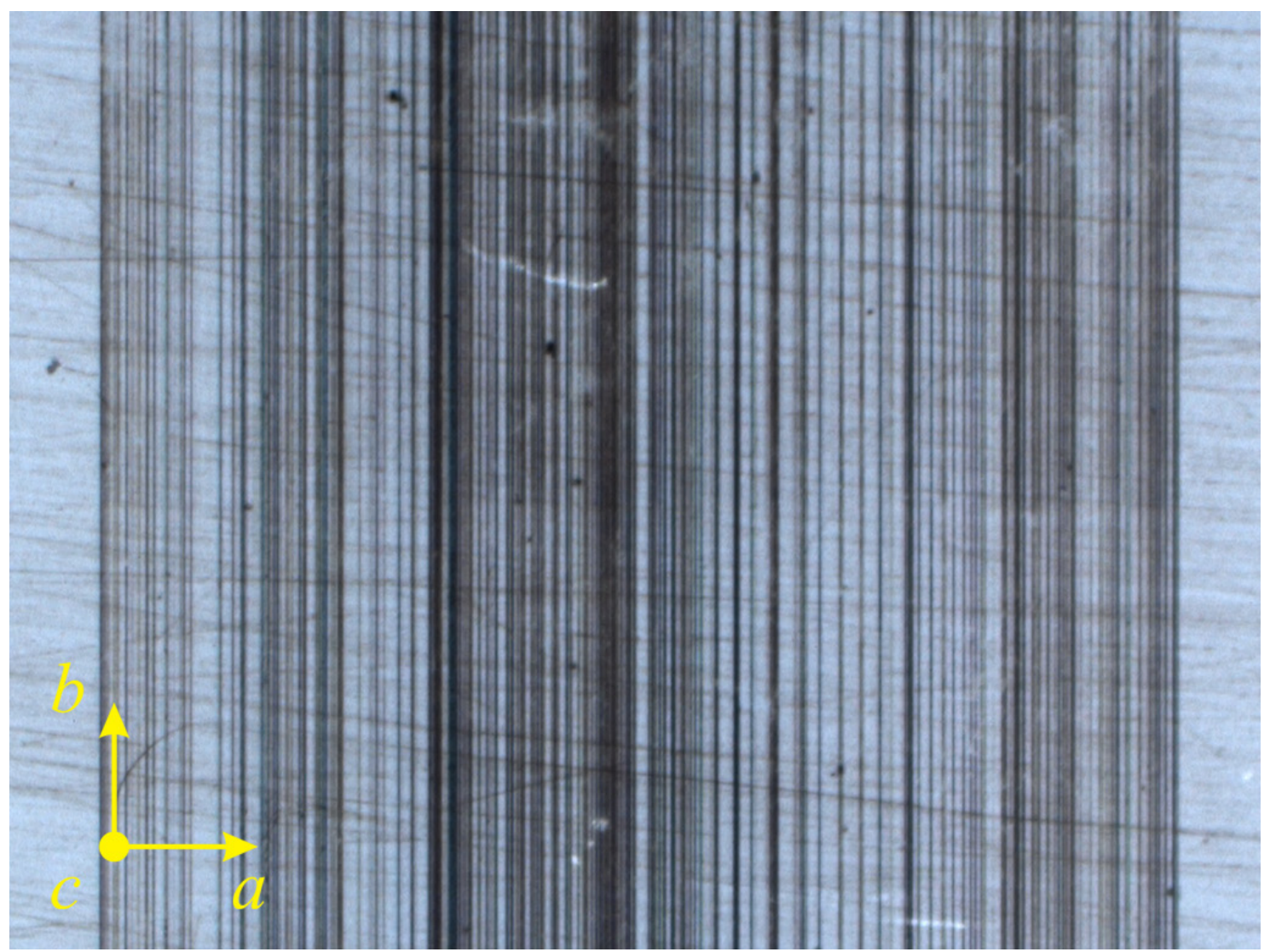

The origin of domains in SBO remains unclear and intriguing from a theoretical point of view. However, starting with experimental facts, we can state that it is connected with an instability of the position of one of ions, most likely strontium, on the interface between growing crystal and the melt. Two possible minima of potential energy probably exist for this ion, depending on the process of boron-oxygen network formation. In completely formed network the position of strontium is strictly determined and if all boron ions occupy proper minima in the process of network formation, then strontium ions are likely to occupy main potential minimum position, and domain-free crystal structure keeps forming. If some boron ions inside incompletely formed network are in the position of secondary minimum, then adjoining strontium ion can occupy its secondary minimum displaced from the main minimum in the direction of $c$ axis. In this case, the seed of an inverted domain forms, and subsequent atomic layers can follow the crystal structure corresponding to inverted orientation of atoms in the unit cell. Such kind of instability is rather common for the formation of defects in the process of growth of various crystals, so we must deduce that in case of SBO this instability leads to rather rare consequence like formation of domain structures. To our knowledge, SBO is the only non-ferroelectric crystal where such domain structures are formed during controlled single crystal growth.

All the samples studied in this paper are obtained via common Czochralski technique. The seed crystal was a domain-free one and was oriented along $b$ axis in the direction of pulling. The starting temperature on the melt surface for all growth experiments was $1000^{\circ} \mathrm{C}$, the seed rotation rate was $10 \mathrm{rpm}$, the pulling rate was $2.4 \mathrm{~mm} /$ day, and the cooling rate was $1{ }^{\circ} \mathrm{C} /$ day. However, since 
microscopic growth instability plays its role in domain formation, minor uncontrollable deviations of local parameters on the surface between the crystal and the melt have led to variation of domain structure and nonlinear optical behavior of the samples obtained in separate growth experiments, as described below.

The dimensions of domain structures obtainable in SBO can be as large as $1 \times 1 \mathrm{~cm}$ in the directions of $b$ and $c$ axes. The thickness of the region containing domains in the direction of $a$ axis can be several millimeters; however, the thickness of individual domains in this direction is highly randomized. The largest domains within the structure can be more than one hundred microns, while smallest detected domains are less than hundred nanometers thick. This explains why RSV spectrum of NPC SBO is smeared over extremely large spectral range with corresponding overall decrease of RSV amplitudes. Hence, possible applications of NPC SBO are different the conversion processes of broadband widely tunable laser sources, preferentially femtosecond ones. For fs pulses, radiation damage threshold becomes higher than in nanosecond pulsewidth scale, and the RSV spectrum smearing can be compensated by fundamental wave intensity. Special interest can be in the creation of widely tunable source over the vacuum ultraviolet.

\section{Applications of NPC SBO}

\subsection{Generation of Deep UV Radiation in NPC SBO}

Existing crystal-based nonlinear frequency converters of laser radiation to the deep and vacuum ultraviolet employ angular phase matching [14]. This kind of phase matching commonly provides the highest efficiency; however, typically it requires a sum frequency conversion scheme with additional tunable parametric oscillator. Generated power strongly depends on the propagation angle, which must be accurately tuned in accordance with the tuning of input laser frequencies. The effective nonlinear coefficient typically decreases with the tuning, as well. All these restrictions limit the spectral region of tuning obtainable in a single nonlinear crystal converter. The features of random NPC converters are quite different, as was established in [10] as regards frequency doubling of narrowband nanosecond pulses. Radiation from a Nd:YAG laser (Lagraph LTI-245, pulse duration $15 \mathrm{~ns}$ ) was initially frequency doubled, and unfocused radiation at $532 \mathrm{~nm}$ with divergence $3 \mathrm{mrad}$ was used as fundamental radiation for the next frequency doubling process in NPC SBO. The angular dependence of the second harmonic at $266 \mathrm{~nm}$ for a highly randomized NPC sample consisting of $3 \mathrm{~mm}$ thick domain-free crystal and $2 \mathrm{~mm}$ thick NPC (Figure 2) radically differs from that of a $0.431 \mathrm{~mm}$ thick single domain sample shown in the inset in the identical power scale, both in the amplitude and in the characteristic angular variation scale. One common feature can be found; namely, decreasing of angular width of oscillation on the growth of rotation angle. In both cases the reason for this behavior is the interference of the contributions from different layers (coherent-length-thick ones in case of single domain sample). For a single domain sample, observed angular oscillations are known as Maker fringes. In analogue, angular behavior of second harmonic in NPC can be considered as "Random Maker fringes" (RMF). They are strongly distorted in amplitude and angular shape reflecting RSV spectrum in NPC. Upon tuning the wavelength of fundamental, RMF will behave similar to common Maker fringes, i.e., minimum at zero angle will turn to maximum and so on (Figure 3). 
Figure 2. (a) Angular dependence of the second harmonic for nonlinear photonic crystals (NPC) and strontium tetraborate (SBO); (b) Maker fringes for single domain sample of SBO. Input radiation $(532 \mathrm{~nm})$ direction $k$, polarization of input radiation and second harmonic (266 nm) E.
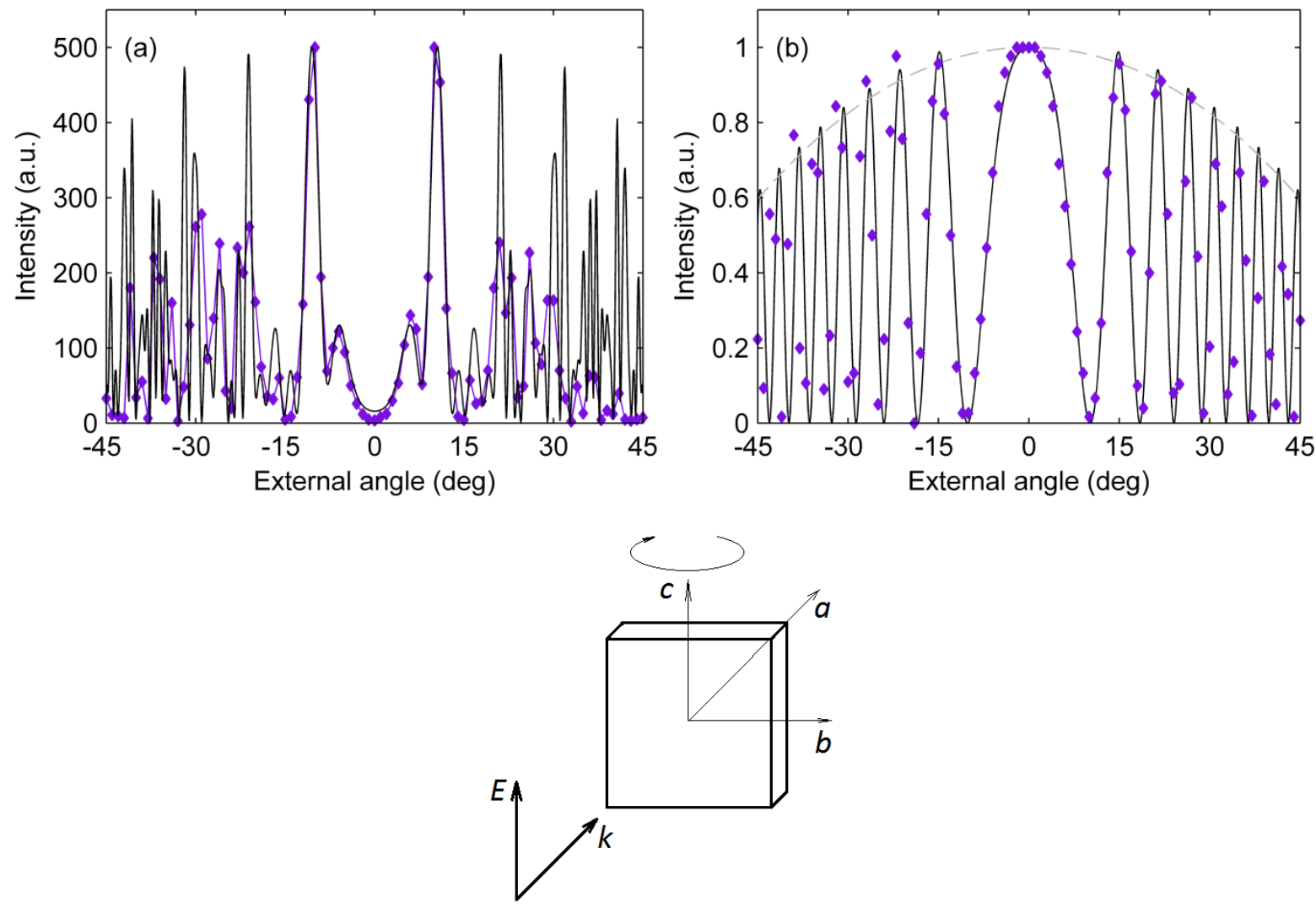

Figure 3. Random Maker fringes for NPC SBO calculated by varying the wavelength.

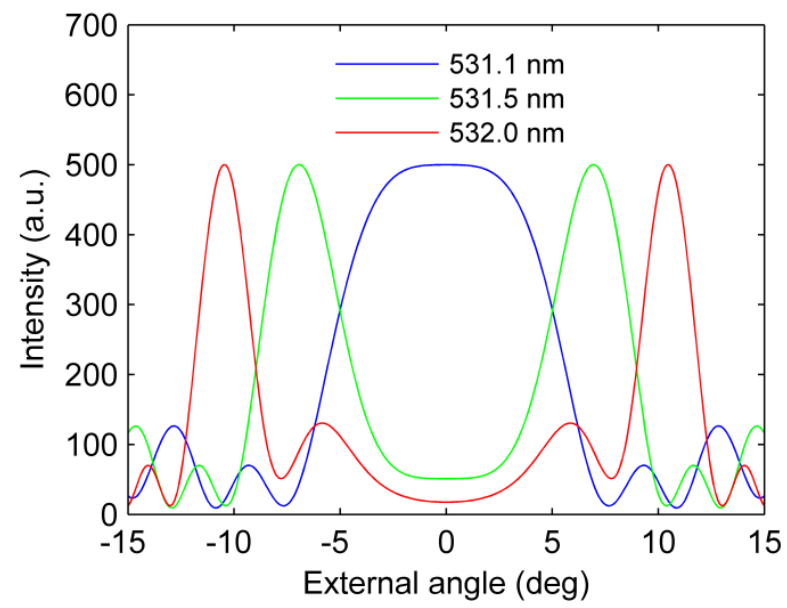

Direct presentation of the RSV spectrum is the dependence of the second harmonic on the fundamental wavelength (Figure 4). This graph clearly demonstrates the result of excessive randomization that leads to a number of relatively narrow peaks covering large spectral area. Such properties are hardly the best fit for any definite application. However, a number of nonlinear optical processes can be enhanced in the same NPC sample. All our samples show experimentally verified enhancement of very different nonlinear optical processes, both for widely tuned infrared and 
ultraviolet fundamental wavelengths. For case of infrared fundamental wavelengths enhancement of efficiency is observed in the same sample for both RQPM and NLD geometries. Enhancement of NLD requires thinner domains in NPC than RQPM at the same fundamental wavelength. As a result, smaller enhancement is expected for ultraviolet fundamental wave in NLD than in RQPM.

Figure 4. Spectral dependence of the second harmonic for a sample of NPC SBO (blue) and single domain sample (black) calculated by varying the fundamental wavelength. Real domain structure of NPC SBO mapped via optical microscopy was used in the calculation.

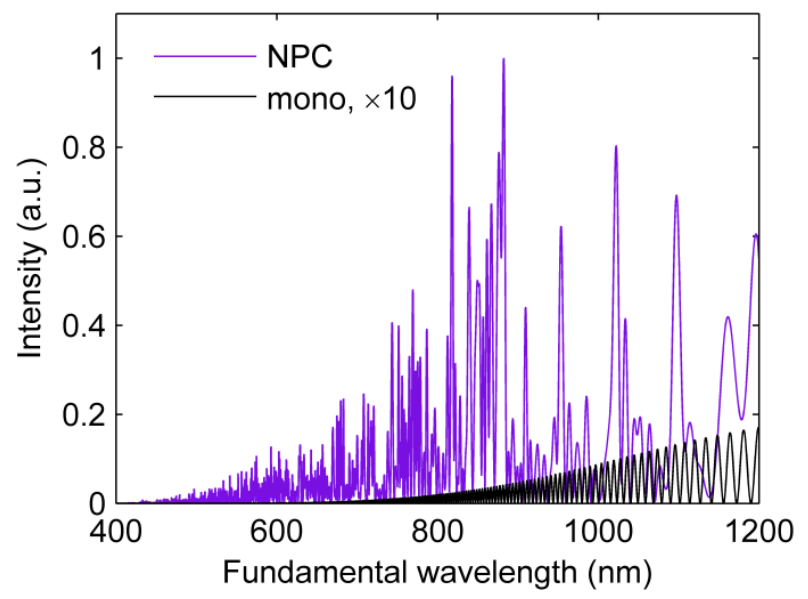

Tunable generation of DUV in NPC SBO was investigated [19] using a femtosecond oscillator (Spectra Physics Tsunami, pulse duration 40-90 fs) of the lowest power class as the starting source of radiation. At the first stage, radiation was converted into the second harmonic using a traditional angularly phase matched BBO converter. The spectrum of the second harmonic pulses was smooth with a width of $2 \mathrm{~nm}$. This radiation with an average power up to $135 \mathrm{~mW}$ was focused into the NPC SBO with the intensity up to $300 \mathrm{MW} / \mathrm{cm}^{2}$ in the direction of RQPM. The doubling of the frequency of input radiation in NPC SBO resulted in generation of DUV in the region of $200 \mathrm{~nm}$. The spectra of DUV were not smooth but contained multiple peak structures with the characteristic width of individual peak in the order of several tenths of a nanometer (Figure 5). These structures are found to be independent of the power of input radiation and therefore they cannot be due to any process of the nonlinearity higher than the second one. Obviously, they are the manifestation of the multiple peak structure seen in the spectrum in Figure 4. Different panels in Figure 5 show spectra obtained in the NPC samples from different growth experiments. These samples had different domain structures but the control of this structure by the growth conditions is presently above our experimental abilities. The quality of spectra is not perfect, but comparison of spectra from samples S2 and S4, from one side, and those from samples S7 and S8, from the other side, show that the degree of spectra deterioration can be in principle controlled by the growth process of SBO crystals using more advanced growth installations. From Figures 4 and 5, one can expect that DUV tuning curve might exhibit strong influence of the same multipeak structure. However, for typical bandwidth of input femtosecond pulse and NPC structure typical for SBO it was found that it is not so. 
Figure 5. The spectra of deep ultraviolet (DUV) generated in several samples (S2, S4, S7, S8) obtained in separate growth experiments.
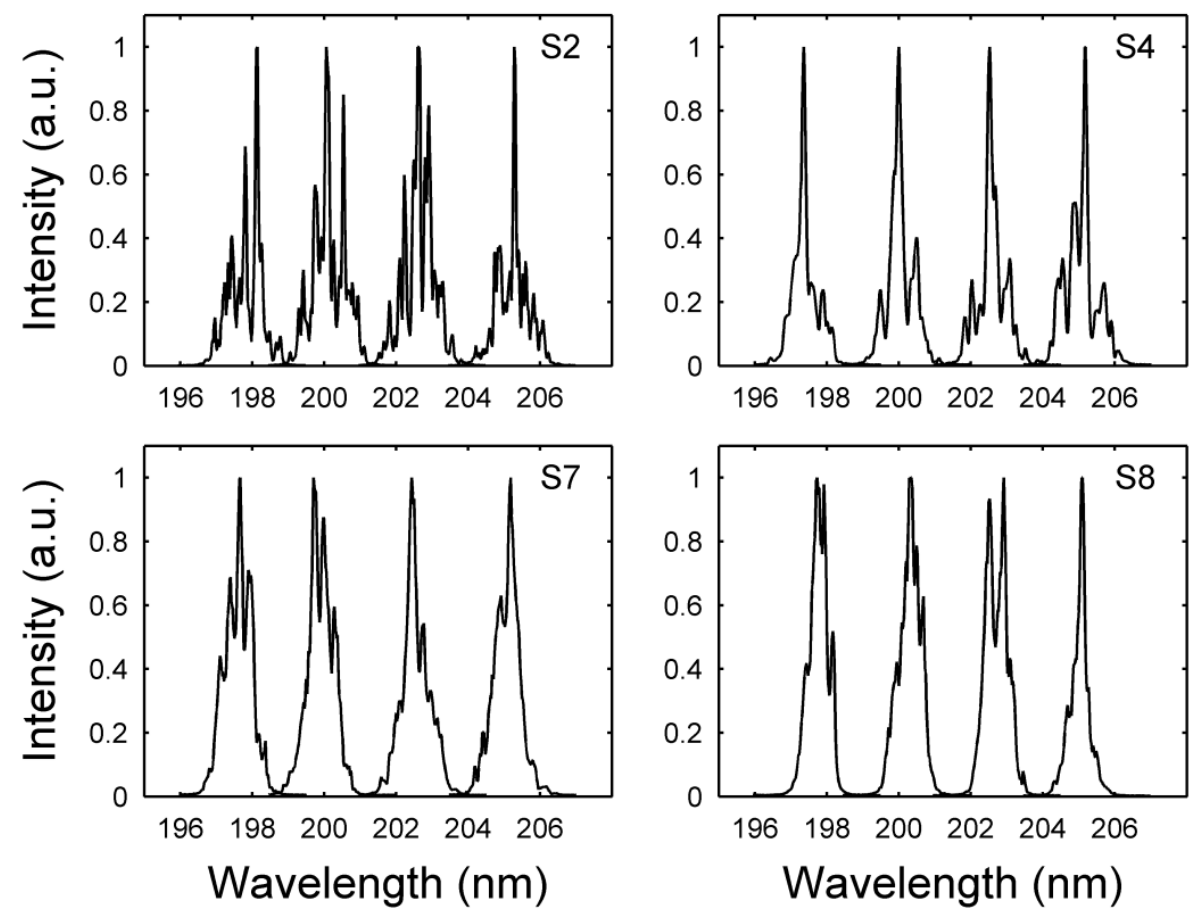

DUV tuning curves (Figure 6) were obtained for several samples at the fixed position of the NPC by a tuning oscillator and BBO doubler. Continuous tuning can typically be obtained in the range from 232.5 to $187.5 \mathrm{~nm}$. The upper limit of this range could be easily shifted approximately to $250 \mathrm{~nm}$, in view of the Tsunami tuning range. The lower limit was determined by the absorption in the air and surely can be extended to shorter wavelengths, probably below $180 \mathrm{~nm}$, since no signs of absorption in NPC material were found. Among investigated samples, there is considerable difference in the efficiency in the order of ten that must be connected with the variativity of as-grown NPCs. Maximum generated power at $200 \mathrm{~nm}$ in the order of $1 \mu \mathrm{W}$. The dependence of generated power on the input power was experimentally found to be quadratic up to an input intensity of $300 \mathrm{MW} / \mathrm{cm}^{2}$ [20], so to increase generated power by $10-100$ is expected in view of the high radiation breakdown threshold of SBO. The decrease of generated power at shorter wavelength is mainly due to air absorption. The decrease of RSV amplitudes is present in this spectral region too, but it plays a secondary role. The tuning curve bears no signs of narrow multiple peak structure in Figure 5, but variations in RSV amplitudes at the scale of several nanometers are well observable. 
Figure 6. DUV tuning curves for several samples (S2, S4, S7, S8) obtained in separate growth experiments. DUV power is normalized to the square of the power of the fundamental beam.

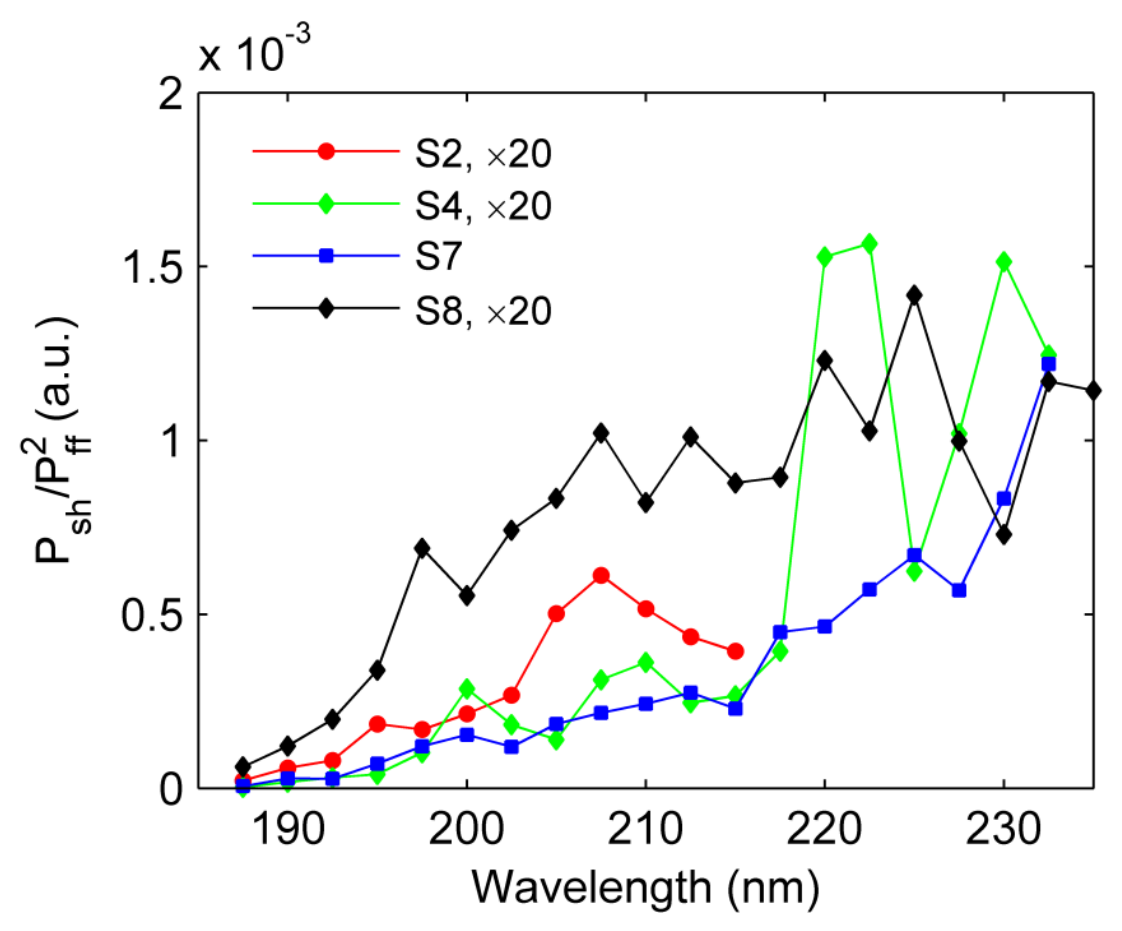

Spectral and angular dependences of generated power in NPCs are connected through the concept of the NPC band structure [10,21]. Peaks observed in both dependences can be associated with the same set of RSV. An example of angular dependence of DUV power is presented in Figure 7. This curve is in general terms symmetrical with respect to the case of normal incidence, deviations from this symmetry being due to the instability of some experimental parameters, i.e., the temperature fluctuations. The range of power variation in the order of three within 30 degrees can be considered as super-critical angular sensitivity of RQPM. Similarly, slow quasi-monotonic decrease of generated power with its central wavelength can be treated as super-critical spectral sensitivity of RQPM.

Figure 7. An example of angular dependence of DUV generated in NPC SBO.
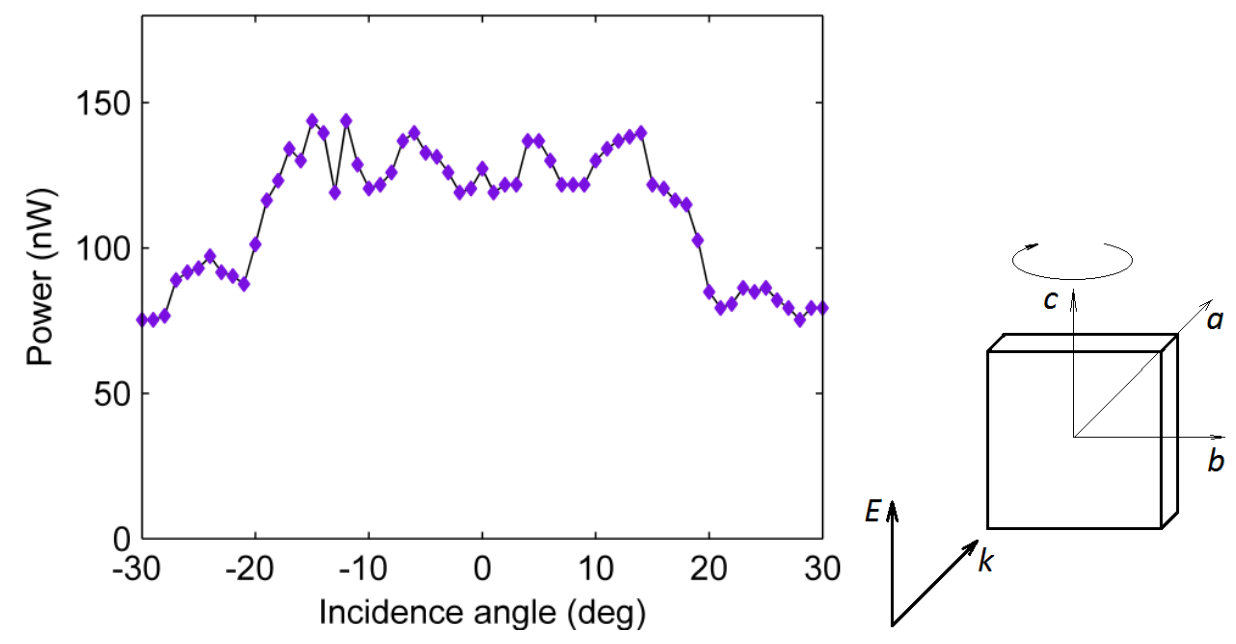


\subsection{Fs Pulses Diagnostics Using NPC SBO}

Pulse diagnostic experiments were conducted for fundamental radiation of the Ti:sapphire laser, in order to investigate all the details of NPC SBO operation as the nonlinear material for these purposes $[22,23]$. A common autocorrelation optical scheme was used to form two intersecting beams of the diagnosed radiation with controllable delay between pulses. In this geometry, both collinear and non-collinear second harmonic generation takes place, but only non-collinear interactions are useful for pulse diagnostics. For non-collinear interactions, both RQPM and NLD geometries are usable for diagnostics. In the former case, to ensure a noticeable RQPM effect for NPC SBO samples in our disposal, the bisector of fundamental beams must coincide or be in the vicinity of the perpendicular to the domain walls. For NLD geometry, the bisector of fundamental beams must lie in the plane of domain walls or be in the vicinity of this plane. In these two cases, nonlinear interaction of intersecting beams employs different parts of RSV spectrum, and it is interesting to compare accuracy and signal-to noise ratio for these two possible schemes. A comparison with angular phase matching was done using properly cut BBO reference crystal.

\subsubsection{RQPM Scheme}

The idea to use RQPM in strongly randomized structures may lead to doubts, due to multiple peak structures expected in autocorrelation signal, since it is typically recognized that for accurate measurement all the spectral components must fall into the phase matching width. Typical autocorrelation curves obtained with RQPM scheme and with reference crystal are presented in Figure 8. It demonstrates excellent agreement between the reference crystal and NPC, despite the doubts mentioned above. Measurement of the second harmonic spectra of autocorrelation signal and single beam signal (Figure 9) reveals that the autocorrelation signal has a much wider spectrum than the single beam signal, which favors the accurate determination of the pulse duration. The modification of the spectrum in autocorrelation beam can be explained by the geometry of interaction of two focused intersecting fundamental beams. The signal-to-background ratio in the case of RQPM is found to be in the order of 3000 , while for angular phase matching in BBO reference crystal it is close to 20000. At the same time, the background level in the experiment with reference crystal was much higher than in that with NPC SBO, while in the case of NPC SBO the background noise was determined by the intrinsic noise of detector. Therefore, using the detector with lower intrinsic noise will increase the signal-to-background ratio in the case of NPC SBO. 
Figure 8. RQPM autocorrelation trace of Tsunami oscillator measured with NPC SBO (blue) and BBO (black). Central wavelength $780 \mathrm{~nm}$. SBO: pulse duration $83.3 \mathrm{fs}$; power 3.49 microwatts; SNR-2992; BBO: pulse duration 83.7 fs; power 929.9 microwatts; SNR-15079.

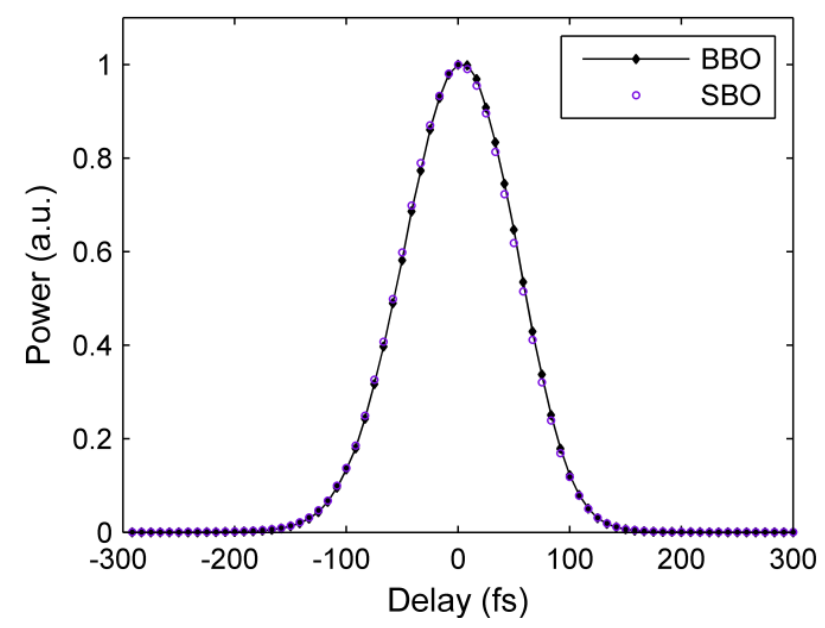

Figure 9. Spectra of autocorrelation signal (a) and single-beam second harmonic (b).
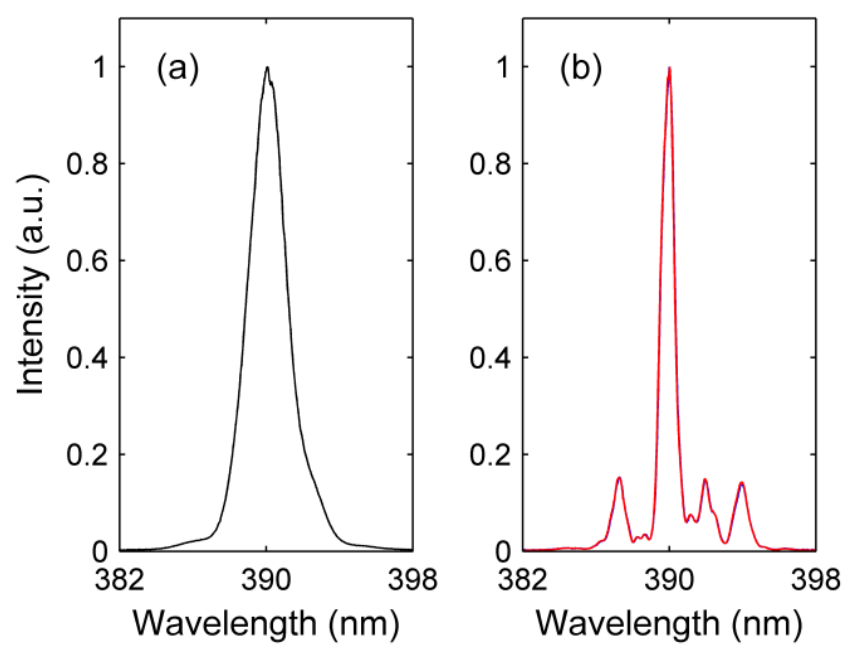

The dependence of pulse duration on the oscillator central wavelength (Figure 10) was obtained throughout all available tuning range of Ti:sapphire oscillator 710-930 nm at fixed position of NPC. Signal-To-Background ratio was in the order of several thousand for all measured points. This admits unique spectral insensitivity of random NPC already pointed out in the case of DUV generation experiments. NPC structures in SBO are known to be highly randomized in $a$ axis direction. Due to this fact, one may expect that the displacing the NPC along this axis may influence accuracy of measurements. Figure 11 presents the results of the spectral measurement of autocorrelation beam obtained in the course of scanning the NPC along the $a$ axis, while all the rest parameters of the optical scheme remained unchanged. Really, the variation in the spectra is rather noticeable. However, autocorrelation traces taken in three positions of NPC indicated by vertical dashes show the same results for the pulse duration. Additionally, this measurement reveals evident "wavy" spatial variation of the NPC structure that arises from some morphological inhomogeneity associated with the growth process of strontium tetraborate crystal sample under study. 
Figure 10. Pulse duration of Tsunami oscillator measured with NPC SBO throughout the tuning range at fixed angular position of the crystal.

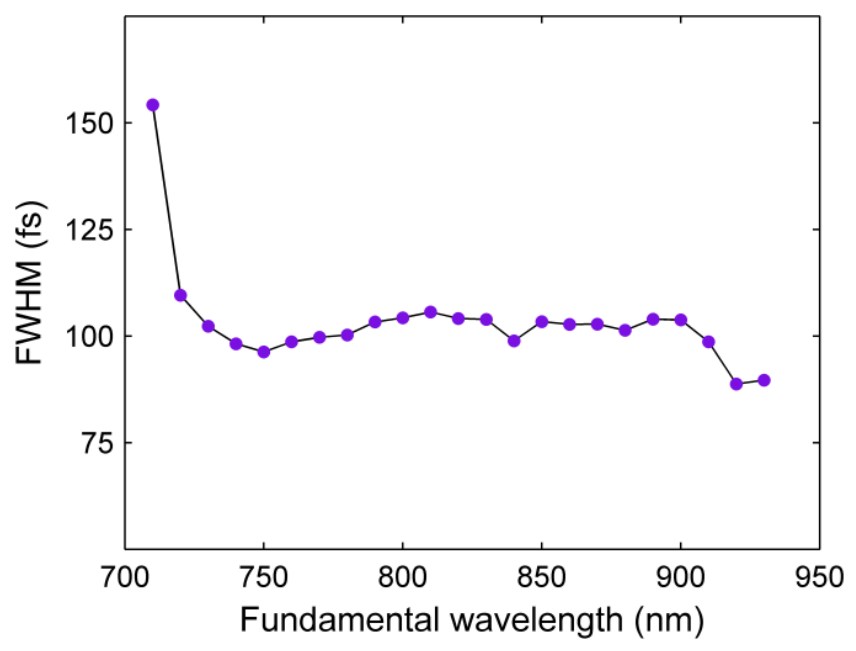

Figure 11. Variation of autocorrelation beam spectra on the NPC position. (a) Net data; (b) Data normalized to the maximal value in every section of constant coordinate.

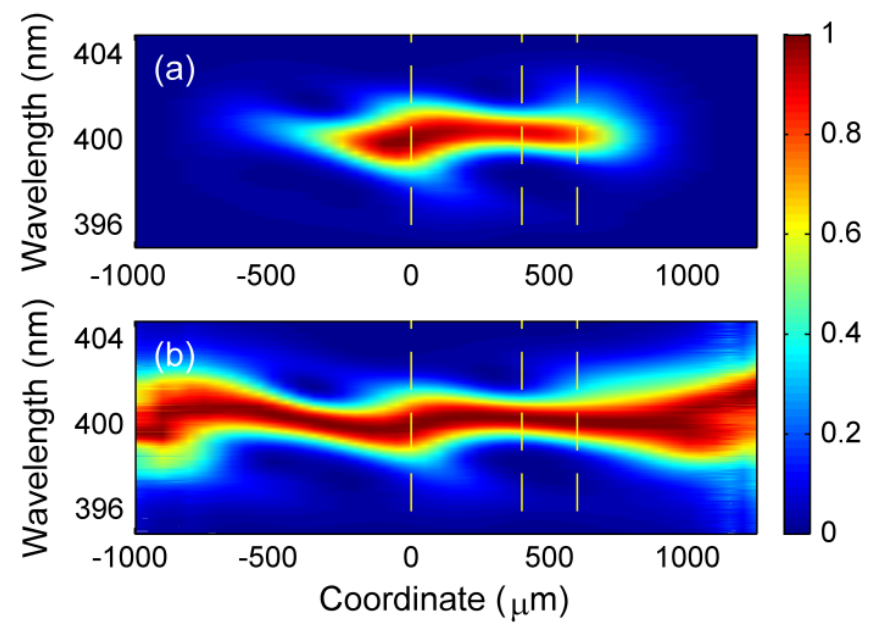

\subsubsection{Autocorrelation Measurements Using Nonlinear Diffraction from Virtual Beam}

Nonlinear diffraction from virtual beam (NLDVB) was recently demonstrated [24]. Nonlinear diffraction from virtual beam is attractive for autocorrelation measurements since the spectral scale of signal variation in the NLD second harmonic generation is larger than within RQPM for typical NPC SBO structures. Depending on the mutual orientation of domain walls plane and beams intersection plane, two different patterns can be obtained in 1D NPC. When these planes are parallel, then the autocorrelation beam does not coincide with any other beams and is always observed separately. When aforementioned planes are perpendicular as in case of normal incidence, there is an overlap of the autocorrelation beam and one of the beams diffracted from a single fundamental beam. In SBO these schemes employ nonlinear coefficients $d_{c a a}$ and $d_{c c c}$, respectively. In view of larger value of $d_{c c c}$, the latter scheme must be more efficient and enable larger dynamic range of measurements. To avoid beam overlapping, NPC must be rotated off the normal incidence position around the axis perpendicular to the intersection plane. This rotation does not affect the effective nonlinear coefficient 
that remains to be equal to $d_{c c c}$. The angle of rotation can be found experimentally from the considerations of minimizing the overlap contribution to the background and maximum contribution of RSV spectrum. Sufficient rotation angle value for our samples was found to be of below ten degrees. The scheme of experimental set-up is presented in Figure 12. Typical autocorrelation curve is presented in Figure 13 in comparison with reference crystal. The agreement is as good as in the case RQPM scheme. The background-to noise ratio was 1600 , that is approximately two times smaller than in RQPM geometry. This difference, in our opinion, is not fundamental and can be decreased by careful adjustment of the experimental setup. In case of NLDVB, the propagation angle of autocorrelation beam changes when tuning the central frequency of the fundamental. The autocorrelation measurements in the course of tuning the central frequency of the fundamental radiation are, therefore, slightly more complicated since it is necessary to monitor this change, but for large aperture detector this difficulty is not severe.

Figure 12. Optical scheme of autocorrelation measurement in nonlinear diffraction from virtual beam (NLDVB) geometry. 1-Tsunami oscillator; 2-beamsplitter; 3-mirror; 4-delay line; 5-focusing lens; 6-NPC SBO; 7-BG39 filter; 8-918D-UV-OD3 sensor.

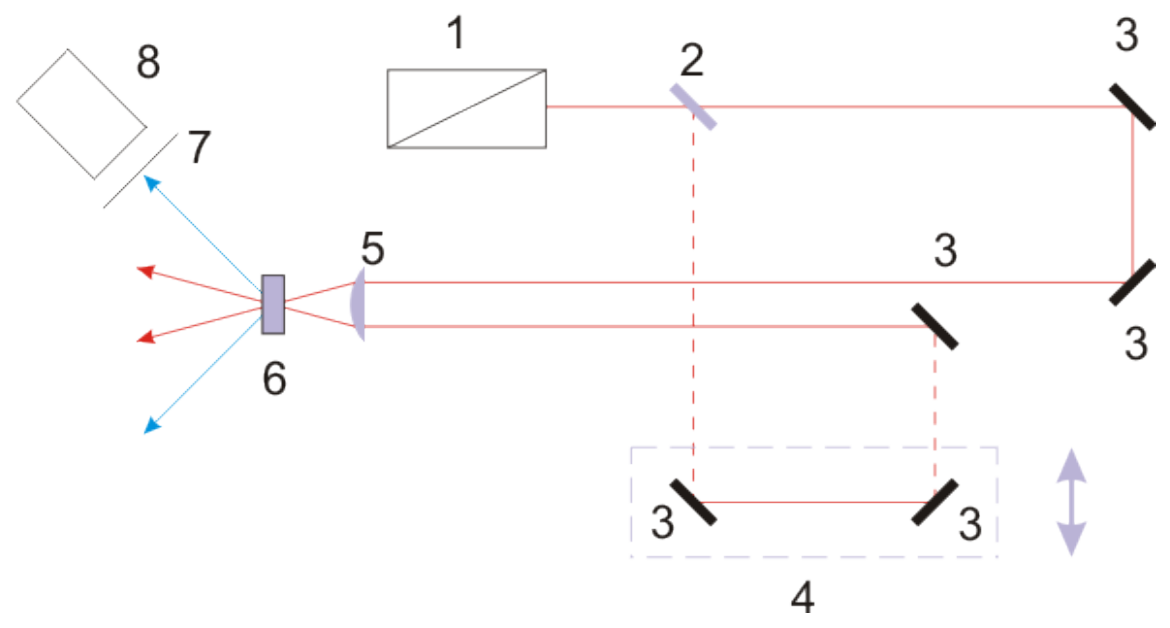

Figure 13. NLDVB autocorrelation trace of Tsunami oscillator measured with NPC SBO (blue) and BBO (black). Central wavelength $840 \mathrm{~nm}$. SBO: pulse duration $75.7 \mathrm{fs}$; power 5.99 microwatts; SNR-1877; BBO: pulse duration 77.0 fs; power 770 microwatts; SNR-17510.

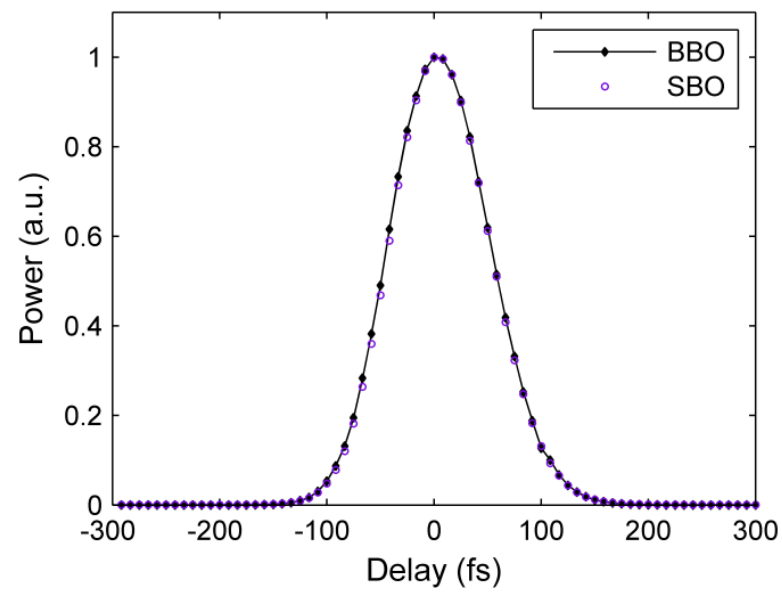




\section{Conclusions}

An overview of recent results on properties and nonlinear frequency conversion processes in NPC SBO is presented. Angular dependence of nonlinear optical conversion efficiency is treated like "random Maker fringes". The variativity of NPC structures obtainable in SBO indicates that spectra of generated radiation can be controlled in the process of NPC growth. Both RQPM and NLDVB schemes are shown to be equally useful for fs pulse diagnostics. Spatial distribution of RSV in NPC is revealed by investigations of generated spectra. Random nonlinear photonic crystals of strontium tetraborate are found to be useful for two applications, namely, generation of DUV radiation and autocorrelation measurements. Nonlinear optical converters based on NPC SBO are featured by unique tolerance to spectral and angular detuning. Due to transparency in the VUV, NPC and SBO are prospective for creation of widely tunable femtosecond source in this spectral region.

\section{Acknowledgments}

The work was supported by the Ministry of Education and Science of Russian Federation (Contracts 16.740.11.0150 and 14.B37.21.0730, and Grant 2.1.1/3455), by RFBR Grant 12-02-31167, by the Russian President Grant SS-1044.2012.2, by RAS Projects 29 and 31, by PSB RAS Projects 2.5.2 and 3.9.5, by SB RAS Projects 43 and 101, and by SFU Grant F12.

Conflict of Interest

The authors declare no conflict of interest.

\section{References and Notes}

1. Joannopoulos, J.D.; Meade, R.D.; Winn, J.N. Photonic Crystals; Princeton University Press: Princeton, NJ, USA, 2008; p. 286.

2. Berger, V. Nonlinear photonic crystals. Phys. Rev. Lett. 1998, 81, 4136-4139.

3. Armstrong, A.; Bloembergen, N.; Ducuing, J.; Pershan, P.S. Interactions between light waves in a nonlinear dielectric. Phys. Rev. 1962, 127, 1918-1939.

4. Fejer, M.M.; Magel, G.A.; Jundt, D.H.; Byer, R.L. Quasi-Phase-Matched second harmonic generation: Tuning and tolerances. IEEE J. Quant. Electron. 1992, 28, 2631-2654.

5. Arie, A.; Voloch, N.C. Periodic, quasi-periodic and random quadratic nonlinear photonic crystals. Laser Photon. Rev. 2010, 4, 355-373.

6. Baudrier-Raybaut, M.; Haidar, R.; Kupecek, P.; Lemasson, P.; Rosencher, E. Random quasi-phase-matching in bulk polycrystalline isotropic nonlinear materials. Nature 2004, 432, 374-376.

7. Morozov, E.Y.; Chirkin, A.S. Stochastic quasi-phase matching in nonlinear-optical crystals with an irregular domain structure. Quantum Electron. 2004, 34, 227-232.

8. Fischer, R.; Saltiel, S.M.; Neshev, D.N.; Krolikovski, W.; Kivshar Y.S. Broadband femtosecond frequency doubling in random media. Appl. Phys. Lett. 2006, 89, 1-3. 
9. Aleksandrovsky, A.S.; Vyunishev, A.M.; Zaitsev, A.I.; Zamkov, A.V.; Arkhipkin, V.G. Detection of randomized nonlinear photonic crystal structure in a non-ferroelectric crystal. J. Opt. A, Pure Appl. Opt. 2007, 9, S334-S338.

10. Aleksandrovsky, A.S.; Vyunishev, A.M.; Shakhura, I.E.; Zaitsev, A.I.; Zamkov, A.V. Random quasi-phase-matching in nonlinear photonic crystal structure of strontium tetraborate. Phys. Rev. A 2008, 78, 31802-31806.

11. Shutov, I.V.; Chirkin, A.S. Consecutive high-order harmonic generation and formation of subfemtosecond light pulses in aperiodical nonlinear photonic crystals. Phys. Rev. A 2008, 78, doi: 10.1103/PhysRevA.78.013827.

12. Sheng, Y.; Ma, D.L.; Ren, M.L.; Chai, W.Q.; Li, Z.Y.; Koynov, K.; Krolikowski, W. Broadband second harmonic generation in one-dimensional randomized nonlinear photonic crystal. Appl. Phys. Lett. 2011, 99, doi: 10.1063/1.3614558.

13. Simagina, L.V.; Mishina, E.D.; Semin, S.V.; Ilyin, N.A.; Volk, T.R.; Gainutdinov, R.V.; Ivleva, L.I. Second harmonic generation in microdomain gratings fabricated in strontium-barium niobate crystals with an atomic force microscope. J. Appl. Phys. 2011, 110, doi: $10.1063 / 1.3624800$.

14. Petrov, V.; Rotermund, F.; Noack, F.; Ringling, J.; Kittelmann, O.; Komatsu, R. Frequency conversion of Ti:Sapphire-Based femtosecond laser systems to the 200-nm spectral region using nonlinear optical crystals. IEEE J. Sel. Top. Quantum Electron. 1999, 5, 1532-1542.

15. Kurimura, S.K; Harada, M.; Muramatsu, K.; Ueda, M.; Adachi, M.; Yamada, T.; Ueno, T. Quartz revisits nonlinear optics: Twinned crystal for quasi-phase matching. Opt. Mater. Express 2011, 1, 1367-1375.

16. Petrov, V.; Noack, F.; Shen, D.Z.; Feng, P.; Shen, G.Q.; Wang, X.Q.; Komatsu, R.; Alex, V. Application of the nonlinear crystal $\mathrm{SrB}_{4} \mathrm{O}_{7}$ for ultrafast diagnostics converting to wavelengths as short as $125 \mathrm{~nm}$. Opt. Lett. 2004, 29, 373-375.

17. Zinenko, V.I.; Pavlovskii, M.S.; Zaitsev, A.I.; Krylov, A.S.; Shinkorenko, A.S. Vibrational spectra and elastic piezoelectric and polarization properties of the $\alpha-\mathrm{SrB}_{4} \mathrm{O}_{7}$ crystal. J. Exp. Theor. Phys. 2012, 115, 455-461.

18. Zaitsev, A.I.; Aleksandrovsky, A.S.; Vasiliev, A.D.; Zamkov, A.V. Domain structure in strontium tetraborate single crystal. J. Cryst. Growth 2008, 310, 1-4.

19. Aleksandrovsky, A.S.; Vyunishev, A.M.; Zaitsev, A.I.; Slabko, V.V. Random-Quasi-Phase matched conversion of broadband radiation in a nonlinear photonic crystal. Phys. Rev. A 2010, 82, doi: 10.1103/PhysRevA.82.055806.

20. Aleksandrovsky, A.S.; Vyunishev, A.M.; Zaitsev, A.I.; Ikonnikov, A.A.; Pospelov, G.I.; Rovskii, V.E.; Slabko, V.V. Deep-UV generation in an SBO crystal with an irregular domain structure. Quantum Electron. 2011, 41, 748-753.

21. Aleksandrovsky, A.S.; Vyunishev, A.M.; Shakhura, I.E.; Zaitsev, A.I.; Zamkov, A.V. Nonlinear optical processes in domain structures of strontium tetraborate. Opt. Spectrosc. 2009, 107, 359-362.

22. Aleksandrovsky, A.S.; Vyunishev, A.M.; Zaitsev, A.I.; Ikonnikov, A.A.; Pospelov, G.I. Ultrashort pulses characterization by nonlinear diffraction from virtual beam. Appl. Phys. Lett. 2011, 98, doi: $10.1063 / 1.3554370$. 
23. Aleksandrovsky, A.S.; Vyunishev, A.M.; Zaitsev, A.I.; Pospelov, G.I.; Slabko, V.V. Diagnostics of fs pulses by noncollinear random quasi-phase-matched frequency. Appl. Phys. Lett. 2011, 99, doi: 10.1063/1.3664094.

24. Saltiel, S.M.; Neshev, D.N.; Krolikowski, W.; Voloch-Bloch, N.; Arie, A.; Bang, O.; Kivshar, Y.S. Nonlinear diffraction from a virtual beam. Phys. Rev. Lett. 2010, 104, doi: 10.1103/PhysRevLett.104.083902.

(C) 2012 by the authors; licensee MDPI, Basel, Switzerland. This article is an open access article distributed under the terms and conditions of the Creative Commons Attribution license (http://creativecommons.org/licenses/by/3.0/). 